\title{
The application of GBS markers for extending the dense genetic map of rye (Secale cereale L.) and the localization of the $R f c 1$ gene restoring male fertility in plants with the $C$ source of sterility-inducing cytoplasm
}

\author{
Pawel Milczarski $^{1} \cdot$ Monika Hanek $^{1} \cdot$ Mirosław Tyrka $^{2} \cdot$ Stefan Stojalowski $^{1}$
}

Received: 2 February 2016/Revised: 22 March 2016 / Accepted: 27 March 2016 /Published online: 16 April 2016

(C) The Author(s) 2016. This article is published with open access at Springerlink.com

\begin{abstract}
Genotyping by sequencing (GBS) is an efficient method of genotyping in numerous plant species. One of the crucial steps toward the application of GBS markers in crop improvement is anchoring them on particular chromosomes. In rye (Secale cereale L.), chromosomal localization of GBS markers has not yet been reported. In this paper, the application of GBS markers generated by the DArTseq platform for extending the high-density map of rye is presented. Additionally, their application is used for the localization of the $R f c 1$ gene that restores male fertility in plants with the $\mathrm{C}$ source of sterility-inducing cytoplasm. The total number of markers anchored on the current version of the map is 19 , 081 , of which 18,132 were obtained from the DArTseq platform. Numerous markers co-segregated within the studied mapping population, so, finally, only 3397 unique positions were located on the map of all seven rye chromosomes. The total length of the map is $1593 \mathrm{cM}$ and the average distance between markers is $0.47 \mathrm{cM}$. In spite of the resolution of the map being not very high, it should be a useful tool for further studies of the Secale cereale genome because of the presence
\end{abstract}

Communicated by: Andrzej Górny

Electronic supplementary material The online version of this article (doi:10.1007/s13353-016-0347-4) contains supplementary material, which is available to authorized users.

Stefan Stojałowski

sstojalowski@zut.edu.pl

1 Department of Plant Genetics, Breeding and Biotechnology, West Pomeranian University of Technology in Szczecin, Słowackiego 17, 71-434 Szczecin, Poland

2 Department of Biochemistry and Biotechnology, Rzeszów University of Technology, Powstańców Warszawy 6 , 35-959 Rzeszów, Poland on this map of numerous GBS markers anchored for the first time on rye chromosomes. The $R f c l$ gene was located on highdensity maps of the long arm of the 4R chromosome obtained for two mapping populations. Genetic maps were composed of DArT, DArTseq, and PCR-based markers. Consistent mapping results were obtained and DArTs tightly linked to the $R f c l$ gene were successfully applied for the development of six new PCRbased markers useful in marker-assisted selection.

Keywords Cytoplasmic male sterility (CMS) - Genetic map • Genotyping by sequencing (GBS) $\cdot$ Rye

\section{Introduction}

Rye (Secale cereale L.) is an outbreeding cereal grown in many regions of the world, but it is most popular in Central and Eastern Europe. This is due to its high resistance to the unfavorable environmental conditions frequently occurring in this area, such as sandy, acidic soils, frosty winters, and water deficiencies in spring. Rye is grown in farms applying modern, intense agricultural technologies, but tolerance to soils with a low content of nutrient solutions and moderate susceptibility to plant diseases also makes it very useful for low-input farming. Despite the importance of this crop in numerous European countries, breeding progress in rye is low compared to related cereals, for example wheat and barley. A noticeable acceleration in the development of new cultivars of rye has been achieved during the past three decades via the introduction of hybrid breeding methods (Geiger 2007).

Seed production of rye hybrid cultivars, similar to many other crops, is based on systems of cytoplasmic male sterility (CMS). The first documented case of CMS in rye was described in the United States by Putt (1954), but this source of sterility-inducing cytoplasm was likely not saved. 
Subsequently, beginning in the late 1960s, several reports on CMS in rye have been published (e.g., Kobyljanskij 1969; Geiger and Schnell 1970; Lapiński 1972; Madej 1975; Adolf and Winkel 1985). Currently, based on the results of test crosses (Warzecha and Salak-Warzecha 1996; Lapiński and Stojałowski 2003) and molecular analyses (Stojałowski et al. 2006, 2008), it is assumed that two genetically different cytoplasms inducing male sterility exist in rye. The Pampa sterility-inducing cytoplasm was discovered by Geiger and Schnell (1970) in a primitive Argentinean population, and this type of CMS is now the most significant for the development of hybrid cultivars of rye. The majority of the remaining reported cases of the discovery of CMS sources supposedly revealed the same genetic type of CMS, namely cytoplasm Vavilovii (CMS-V). These are represented by CMS sources designated as CMS-R (Kobyljanskij 1969), CMS-C (Łapiński 1972), CMS-G (Adolf and Winkel 1985), and others.

Nuclear genes that restore male fertility in rye with Pampa and Vavilovii cytoplasms are spread throughout the rye genome; rough mapping analyses have indicated that they are localized on chromosomes 1R, 3R, 4R, 5R, and 6R (Börner et al. 1998; Miedaner et al. 2000; Bednarek et al. 2002; Stojałowski et al. 2004). Among these genes, those most efficient at fertility restoration were located on the long arm of chromosome 4R. Significant progress in mapping the main restorer gene for the Pampa cytoplasm $(R f p 1)$ has been achieved (Stracke et al. 2003; Hackauf et al. 2012); less information is available with respect to CMS-V restorers. Recently, the application of diversity array technology (DArT) for the construction of a high-density consensus map of rye based on recombinant inbred line (RIL) populations (Milczarski et al. 2011) enabled the identification of markers more closely linked to $R f c 1$, the main restorer gene for rye with CMS-C (Stojałowski et al. 2011).

This study was aimed at the application of next-generation sequencing (NGS) technology for genotyping by sequencing (GBS) to expand the genetic map of rye and, finally, localize the $R f c l$ gene with a higher level of precision.

\section{Materials and methods}

\section{Plant material}

For extending the genetic map of seven rye chromosomes, the mapping population "RIL-S" was applied. This population consisted of a set of RILs developed from a cross between two lines advanced in inbreeding: 541 and 2020LM. This mapping population had previously been analyzed during the construction of a high-density consensus genetic map of rye, and detailed information is given by Milczarski et al. (2011). The maternal line of the studied RIL population was a non-restorer for CMS-C, and the parental line 2020LM contained the $R f c l$ gene that restored plant fertility. The test crosses and following phenotyping analyses allowed for an assessment of which RIL carries the dominant restorer alleles and which possesses the recessive non-restorer allele; these analyses were previously described by Stojałowski et al. (2011).

The new mapping population was developed to localize the $R f c l$ gene. The source material for this population was a hybrid between male-sterile inbred line 544C containing the $\mathrm{C}$ cytoplasm and the restorer inbred line Ot0-20. Research conducted on the $\mathrm{F}_{2}$ generation of this hybrid indicated that the paternal line contained at least two effective restorer genes and that one of the supposed genes was located on the 4RL chromosome (Stojałowski et al. 2004). The male-fertile hybrid plants from the $F_{1}$ and following generations were used for consecutive back-crossing with male-sterile line 544C. In the $\mathrm{BC}_{5}$ generation, eight male-fertile plants were selfed. As a result, a $\mathrm{BC}_{5} \mathrm{~F}_{2}$ mapping population consisting of 1194 individuals was obtained. Plants of the mapping population were grown with $25 \times 25 \mathrm{~cm}$ spacing in an experimental field located at Szczecin. Before flowering, 3-5 spikes of each individual plant were isolated with the use of bags made of transparent breathable foil. During flowering, each plant was visually assessed 2-3 times with respect to pollen shedding, and observations were recorded by using the nine-step scale suggested by Geiger and Morgenstern (1975). The correctness of visual observations was verified after harvest by the assessment of seed setting in previously isolated spikes. Individuals with inconsistent phenotyping results were removed from further analyses.

\section{Generation of molecular markers}

Within both studied mapping populations, three methods were applied for the generation of molecular markers:

1. Polymerase chain reaction (PCR): two types of sequencespecific markers were used: sequence characterized amplified regions (SCARs) and simple sequence repeats (SSRs). Analyses of these markers within the RIL-S population had previously been used for the construction of a consensus genetic map, so all important methodological information can be found in Milczarski et al. (2011) and Stojałowski et al. (2009).

2. DArT: the microarray-based analyses were performed as a commercial service offered by Diversity Arrays Technology Pty Ltd. (Canberra, Australia), and the methodological procedure for the development of DArT markers in rye is described by Bolibok-Bragoszewska et al. (2009).

3. DArTseq: the platform developed by Diversity Arrays Technology Pty Ltd. for high-throughput genotyping based on sequencing results generated by NGS 
technologies. This variant of the GBS method was optimized for numerous plant species and is offered as a commercial service by DArT P/L. The principles of this method were recently published by Li et al. (2015). In brief, the crucial point of the analytical procedure is the method of reduction of genome complexity developed initially for the DArT mentioned above. This reduction is achieved by the application of optimized combinations of restriction enzymes. Sequencing analyses are performed on a HiSeq2000 (Illumina, USA), and the results consist of two groups of detected markers based on short DNA sequences. The first group is designed as SNP markers containing detailed data about the type and position of mutations that enable detection of the heterozygotes and both types of homozygotes within the mapping population (socalled co-dominant markers). The second group is called Silico-DArT, whose data inform only about the presence or absence of a given sequence variant (these markers are of dominant character).

\section{Construction of a high-density genetic map of rye}

The maximum level of deviation of the markers from the distribution of 1:1 characteristic of the RIL populations was set at $p=0.005$. After the reduction of markers significantly deviated from the assumed model, those remaining were binned for reducing redundant markers using the QTL IciMapping software (Wang et al. 2014a) separately for Silico-DArT and SNP. Redundant markers are completely correlated in a population and cannot provide additional information. In the next step, markers with missing rates higher than $20 \%$ were deleted. Markers that were selected as representatives of the bin and not assigned to the bins were combined with DArT markers, as described previously by Milczarski et al. (2011). The genetic map construction was conducted using Multipoint 3.2 software (Ronin et al. 2012). The marker groups were formed at a maximum threshold level of recombination frequencies at 0.05 using the "order" command. For testing the reliability of multilocus mapping by detection and removing problematic markers that caused neighborhood instabilities, "control of monotony" was used. Finally, the ordering was repeated.

In the mapping population, we found an increase in the length of genetic maps compared to the original consensus map constructed using low-density genotyping data. The map length for a chromosome with a minimum distance between 1000 markers $\geq 1 \mathrm{cM}$ provides a map of $\sim 1000 \mathrm{cM}$. This is unrealistic in the vast majority of organisms, but it is unlikely to be caused by high genotype calling error rates. Wang et al. (2014b) suggested that it could be a result of the accumulation of low levels of genotyping error in maps which contain many thousands of markers. For reducing the inflation of genetic distances on a high-density genetic map, the average length of the consensus map (Milczarski et al. 2011) was used for the proportional scaling of obtained linkage groups, as previously described by Cavanagh et al. (2013) and Maccaferri et al. (2014).

\section{Comparative mapping and localization of the $R f c 1$ gene on a higher resolution map}

Identification of molecular markers tightly linked to the $R f c l$ gene was performed within both studied mapping populations using JoinMap 3.0 software (Van Ooijen and Voorrips 2001). The data concerning all segregating markers (PCR-based, DArT, DArTseq) were analyzed (redundant markers were not excluded from the analysis). Linkage groups were constructed at $\mathrm{LOD}=20$, and the group containing the $R f c l$ locus was selected for mapping in each population. Linkage maps were constructed for these two groups by using a regression mapping algorithm. Results were compared using MapChart 2.1 software (Voorrips 2002).

In order to increase the precision of mapping of the $R f c 1$ gene, sequencing data about tightly linked DArT and DArTseq markers located on RIL-S and [544 $\times$ Ot0$20] \mathrm{BC}_{5} \mathrm{~F}_{2}$ mapping populations were used for the designation of PCR primers. These primers, as well as previously developed SCAR markers (Stracke et al. 2003; Stojałowski et al. 2011) located in the vicinity of the $R f c 1$ gene, were applied for analyses on an extended number of individuals from the $[544 \times \mathrm{Ot} 0-20] \mathrm{BC}_{5} \mathrm{~F}_{2}$ population. In total, 658 individuals were genotyped to construct a map with increased resolution.

\section{Effectiveness of markers in the selection of non-restorer lines and DNA sequence annotations}

CMS-C is not widely applied for breeding rye hybrids, mainly due to the deficiency of non-restorer lines and their stable male-sterile analogs. Currently, only a few related nonrestorer lines are available for studies. We chose a set of 95 inbred lines developed from a cross between non-restorer 541 and restorer Ot1-3 as plant material for testing the effectiveness of marker-assisted selection (MAS). All these lines were test-crossed and their ability to restore or not restore male fertility in the CMS-C system was determined in field experiments (Stojałowski et al. 2011). PCR-based markers from the above higher resolution map, which revealed polymorphism between 541 and Ot1-3, were applied for genotyping of the 95 studied inbred lines and for the hypothetical selection of nonrestorer lines. The effectiveness of selection was verified using a standard $\mathrm{Chi}^{2}$ test. Markers were considered efficient when the ratio of non-restorer and restorer lines differed significantly from that observed within the total population of studied lines (48:47). 
The known DNA sequences of markers used for mapping the $R f c 1$ gene were functionally annotated by performing a Blast search using Blast2GO 3.0 software (https://www. blast2go.com/) (Conesa et al. 2005). Blast matches were considered significant with $\mathrm{E}$-values $<1 \mathrm{e}^{-3}$ conducted against the non-redundant (nr) NCBI sequence database. Gene Ontology (GO) annotations were performed to retrieve molecular function, biological process, and cellular component terms. The sequences were loaded into the Blast2GO program, and BLAST with a minimum E-value of $10^{-3}$ was performed with the program prior to mapping. The mapping step allowed the annotation of the sequences to the GO database for GO terms.

\section{Results}

\section{Results of high-throughput genotyping methods}

Both mapping populations were investigated by using two high-throughput genotyping technologies: DArT and DArTseq. The results of DArT analyses of the RIL-S mapping population had previously been used for construction of the consensus genetic map of rye (Milczarski et al. 2011). The applied methods of marker generation were very efficient: each type was represented by thousands of markers (Table 1). Among these, significant numbers revealed polymorphisms within the RIL-S population. The most numerous group of markers were the Silico-DArTs. They were almost twice as efficient with respect to the detection of genetic polymorphisms as SNP markers developed by using the same platform (DArTseq). The less numerous group of markers polymorphic within the RIL-S population was obtained with the use of the microarray method named DArT. On the other hand, over 2000 of the markers generated by this method covered the whole rye genome and was sufficient for the development of linkage groups of all seven rye chromosomes.

The level of polymorphism detected within the [544 $\times$ Ot0$20] \mathrm{BC}_{5} \mathrm{~F}_{2}$ population was significantly lower than that observed in RIL-S population (Table 1), which was expected as an effect of several cycles of back-crossing performed during development of the mapping population. In this plant material, the SNP markers obtained from the DArTseq platform were also less numerous than the Silico-DArTs. The classic
DArT technique based on hybridization of microarrays generated in the $[544 \times \mathrm{Ot} 0-20] \mathrm{BC}_{5} \mathrm{~F}_{2}$ population the smallest group of polymorphic markers, similarly to that recorded in RIL-S.

\section{High-density genetic map of rye}

The previously released genetic map of the RIL-S population (Milczarski et al. 2011) was expanded via the addition of two types of newly developed GBS markers: Silico-DArTs and DArTseq SNPs. During the mapping procedure from the group of more than 30,000 polymorphic markers (Table 1), numerous markers were not included into linkage groups. Finally, the current version of the map contains more than 19,000 markers (Table 2). The majority of Silico-DArT and SNP markers revealed identical distributions within the mapping population. These "redundant" markers were located in the same position (the same locus) and were grouped within bins represented on the map by one member of each bin. Finally, almost 3400 loci were revealed on the map (Fig. 1). Chromosomes consist of between 300 and almost 600 loci. When the data from bins are considered, the numbers of markers assigned to chromosomes vary from 1878 on $2 \mathrm{R}$ to 3812 on $6 \mathrm{R}$ (Table 2). The majority of these markers are Silico-DArTs.

Markers on the genetic map are distributed relatively evenly along all seven chromosomes (Fig. 1). The shortest map was constructed for chromosome $2 \mathrm{R}$ (about $200 \mathrm{cM}$ ), and this was also the map with the lowest coverage by marker loci; there are only 322 loci mapped on this chromosome (Table 2). The average distance between loci mapped on $2 \mathrm{R}$ slightly exceeds $0.6 \mathrm{cM}$. Linkage maps of the remaining chromosomes contain at least a hundred more loci, and their lengths vary from $215.5(1 \mathrm{R})$ to $251.40(4 \mathrm{R})$. The longest gap between marker loci on this genetic map of rye is located on the 7R chromosome; it was estimated at $2.26 \mathrm{cM}$ (Table 2).

Detailed data about the constructed genetic map containing positions of marker loci and their segregations within population RIL-S are given in the supplementary material ESM1. Among loci identified during map construction, a group of 1123 (about $33 \%$ ) contained more than one molecular marker and, on the map (Fig. 1), these are represented by only one of the binned markers. Redundant markers revealing
Table 1 Number of markers obtained from diversity array technology (DArT) and genotyping by sequencing (GBS) analyses within the two analyzed mapping populations

\begin{tabular}{|c|c|c|c|c|c|c|}
\hline \multirow{2}{*}{$\begin{array}{l}\text { Population } \\
\text { Type of marker }\end{array}$} & \multicolumn{3}{|c|}{$541 \times 2020$ LM RIL $($ RIL-S) } & \multicolumn{3}{|c|}{$544 \times \mathrm{Ot} 0-20 \mathrm{BC}_{5} \mathrm{~F}_{2}$} \\
\hline & DArT $^{\mathrm{a}}$ & Silico-DArT & SNP & DArT & Silico-DArT & SNP \\
\hline Total number of markers & 11,514 & 39,908 & 14,888 & 3739 & 40,280 & 27,120 \\
\hline Number of polymorphic markers & 2257 & 23,437 & 4417 & 348 & 3215 & 1237 \\
\hline Frequency of polymorphic markers & $19.6 \%$ & $58.7 \%$ & $29.7 \%$ & $9.3 \%$ & $8.0 \%$ & $4.6 \%$ \\
\hline
\end{tabular}

${ }^{a}$ Data from DArT analysis within the RIL-S population have been previously published (Milczarski et al. 2011) 
Table 2 Characteristics of the rye genetic map developed for the RIL-S population

\begin{tabular}{|c|c|c|c|c|c|c|c|c|}
\hline \multirow[t]{2}{*}{ Chromosome } & \multicolumn{5}{|c|}{ Number of markers assigned to chromosomes } & \multirow{2}{*}{$\begin{array}{l}\text { The length of } \\
\text { the map }(\mathrm{cM})^{\mathrm{b}}\end{array}$} & \multirow{2}{*}{$\begin{array}{l}\text { Average distance } \\
\text { between loci }(\mathrm{cM})\end{array}$} & \multirow{2}{*}{$\begin{array}{l}\text { Max. distance } \\
\text { between loci }(\mathrm{cM})\end{array}$} \\
\hline & DArT & Silico-DArT ${ }^{\mathrm{a}}$ & $\mathrm{SNP}^{\mathrm{a}}$ & PCR-based & Total $^{\mathrm{a}}$ & & & \\
\hline $1 \mathrm{R}$ & 131 & $1970(363)$ & $421(83)$ & 5 & $2527(581)$ & 215.50 & 0.371 & 1.41 \\
\hline $2 \mathrm{R}$ & 70 & 1605 (194) & $202(57)$ & 1 & $1878(322)$ & 199.90 & 0.621 & 2.02 \\
\hline $3 \mathrm{R}$ & 165 & $2463(272)$ & $207(53)$ & 0 & $2835(490)$ & 225.20 & 0.460 & 1.53 \\
\hline $4 \mathrm{R}$ & 168 & $1816(357)$ & $273(61)$ & 7 & $2264(593)$ & 251.40 & 0.424 & 1.74 \\
\hline $5 \mathrm{R}$ & 112 & $1763(242)$ & $293(69)$ & 0 & $2168(423)$ & 224.30 & 0.530 & 1.88 \\
\hline $6 \mathrm{R}$ & 180 & $3022(270)$ & $606(98)$ & 4 & $3812(552)$ & 240.20 & 0.435 & 2.03 \\
\hline $7 \mathrm{R}$ & 102 & $2632(275)$ & $859(55)$ & 4 & 3597 (436) & 236.50 & 0.542 & 2.26 \\
\hline Total & 928 & $15,271(1972)$ & $2861(476)$ & 21 & $19,081(3397)$ & 1593.00 & 0.469 & 2.26 \\
\hline
\end{tabular}

${ }^{\text {a }}$ In brackets: markers shown on the genetic map of RIL-S (number of loci located on the map), remaining markers were included into bins and are not visualized on the map (these are localized within the loci represented by one marker from each bin)

${ }^{\mathrm{b}}$ The length of the recalculated map (as described in the Materials and methods section; more information is available in Supplementary Table ESM1)

polymorphisms, but not shown on the final map (included into bins), are listed in the ESM2 file. The distribution of binned loci containing more than one molecular marker is shown in Fig. 2. Two bin loci carrying the most numerous groups of markers (642 and 527) were found near each other, and they were mapped onto the proximal part of the $7 \mathrm{R}$ chromosome (Fig. 2). The largest bins located on the remaining chromosomes contained between 74 (5R) and 399 (1R) markers. In general, the location of loci abundant in co-segregating markers combined into bins seems to be randomly distributed: bins containing numerous markers could be found on proximal and distal parts of all rye chromosomes.

\section{Localization of the $R f c l$ gene and predicted efficiency of new markers for the selection of non-restorer lines}

Phenotypic assessment within the studied population of [544 $\times \mathrm{Ot} 0-20] \mathrm{BC}_{5} \mathrm{~F}_{2}$ revealed a bimodal distribution of the trait. The largest groups, with more than 300 individuals, were those classified as partially male-fertile: 5 and 6 on the Geiger and Morgenstern (1975) scale. The next numerous classes, 1 and 2, include male-sterile plants (Fig. 3). When the whole population was divided into two main phenotypic classes, male-sterile (1-3 on the scale) and male-fertile (4-9 on the bonitation scale), the obtained amounts of individuals, $327: 897$, did not differ significantly from the expected $1: 3$ ratio $\left(\mathrm{Chi}^{2}=3.63\right)$, likely indicating monogenic control of male fertility restoration.

One restorer gene was also found when the RIL-S population was studied. Phenotypic data have been published previously (Stojałowski et al. 2011), and the $R f c 1$ gene was located on the genetic map of the long arm of chromosome 4R, at the $221 \mathrm{cM}$ position (Fig. 1).

In the population [544C $\times \mathrm{Ot} 0-20] \mathrm{BC}_{5} \mathrm{~F}_{2}$, four groups of linked markers were identified at $\mathrm{LOD}=20$ (for details, see the ESM3 file). On the basis of the aforementioned map of the RIL-S population, these linkage groups were assigned to fragments of chromosomes 2R, 4R, 6R, and 7R. The most numerous group of markers was identified as a part of the $4 R$ chromosome and it contained the $R f c l$ locus.

In order to increase the precision of mapping the part of the 4RL chromosome containing the $R f c l$ gene, the groups of markers tightly linked with this gene were selected for both analyzed populations: RIL-S and [544 $\times \mathrm{Ot0}-20] \mathrm{BC}_{5} \mathrm{~F}_{2}$. The groups used for map construction consisted of 154 and 170 loci, respectively. Twenty-three markers present in these linkage groups were common for both populations: 21 DArTs and two SCARs (Xscsz23L500 and Xscsz670L900). No common marker from the DArTseq platform was identified.

For the construction of linkage maps covering the region of the genome carrying the $R f c l$ gene, all markers from the aforementioned linkage groups were applied (including cosegregating markers that, during development of the whole genetic map of the RIL-S population, were combined into bins). Loci identified in the RIL-S population are distributed within a distance of $11 \mathrm{cM}$ (Fig. 4), and the $R f_{c l} l$ gene is located in the central part of this short linkage group between the DArT marker XrPt389652 and the SCAR marker Xscsz23L500. The map created for the second population covers a longer fragment of the 4R chromosome; markers are distributed along a distance of $43.5 \mathrm{cM}$ (Fig. 4). Here, the $R f c l$ gene is also located in the middle part of the linkage map (position $25 \mathrm{cM}$ ), near markers Xscsz23L500 (SCAR) and 5213528_68:G>C (SNP).

On the basis of available DNA sequences related to DArT and DArTseq markers tightly linked with $R f c l$, a set of 15 and 16 primer-pair combinations were designed for the development of PCR-based markers (Table ESM4). Of these, only six revealed polymorphism within the mapping population [544 $\times$ Ot0-20]BC ${ }_{5} \mathrm{~F}_{2}: \mathrm{XrPt}_{400382,} \mathrm{XrPt411156,} \mathrm{XrPt505373,}$ 


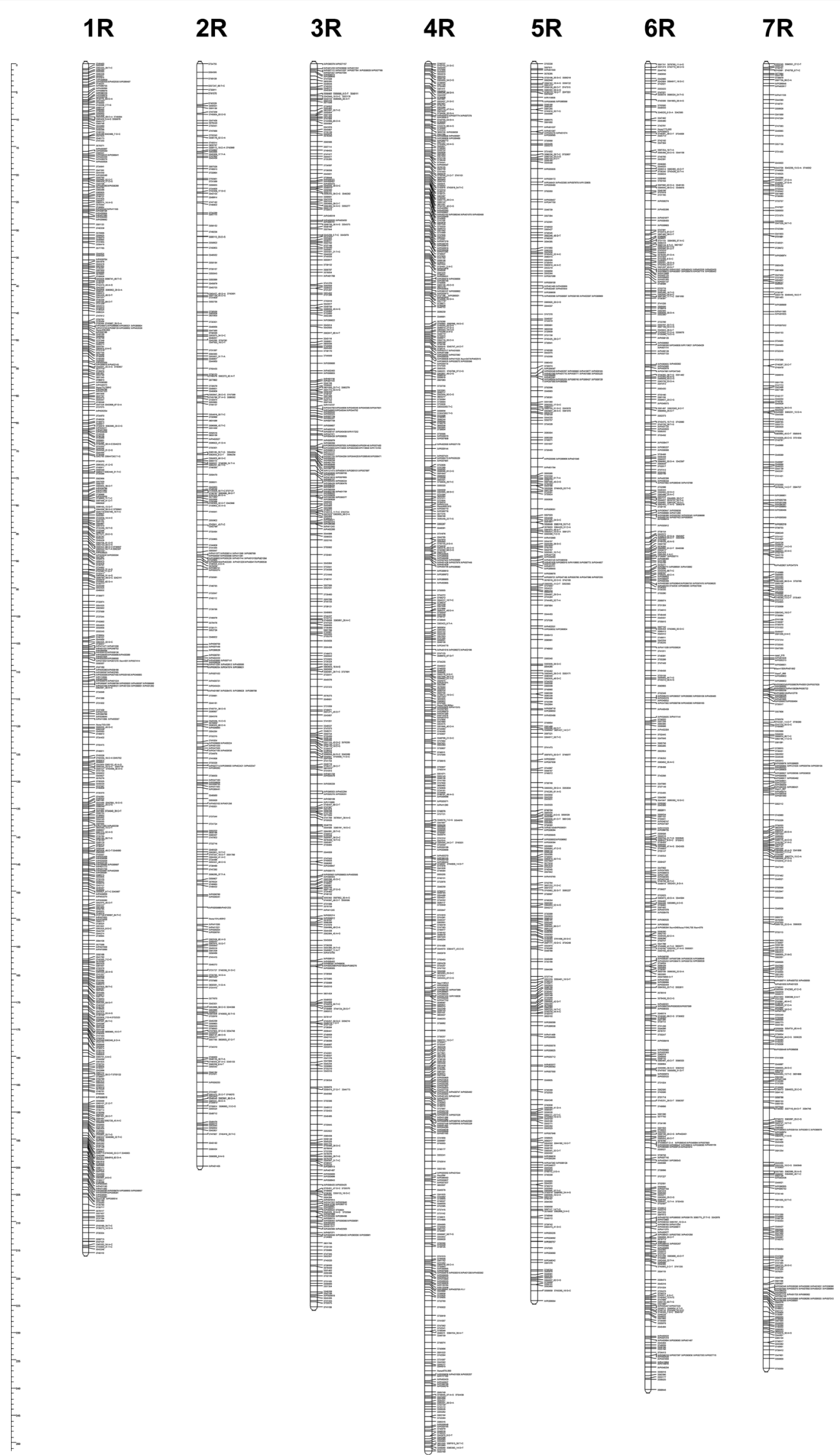

Fig. 1 Genetic map of the RIL-S population (the scale on the left margin shows distances in cM) 
Fig. 2 Distribution of markers within bins located on all rye chromosomes. The $x$-axis shows cumulative distance on the chromosome. The $y$-axis presents the number of markers included in the bin
$1 \mathrm{R}$

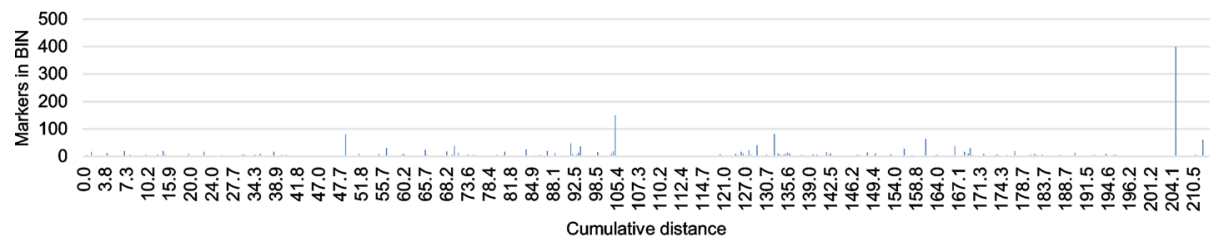

2R

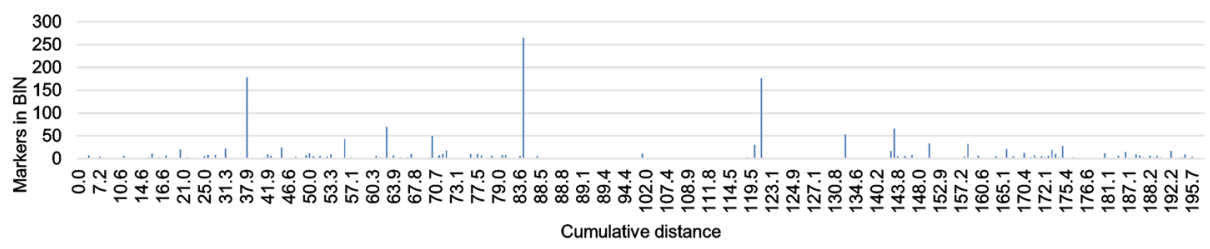

3R

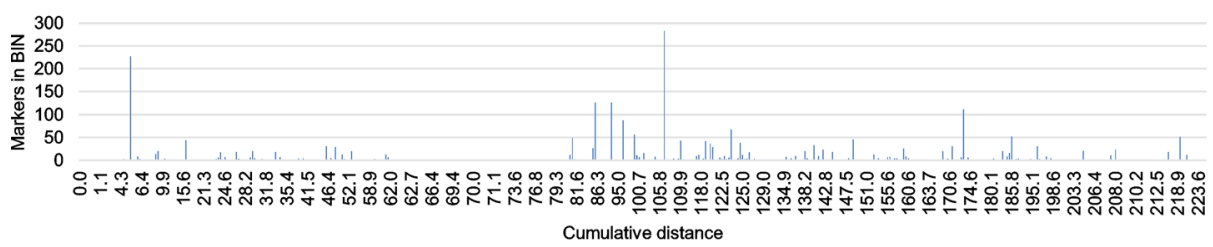

4R

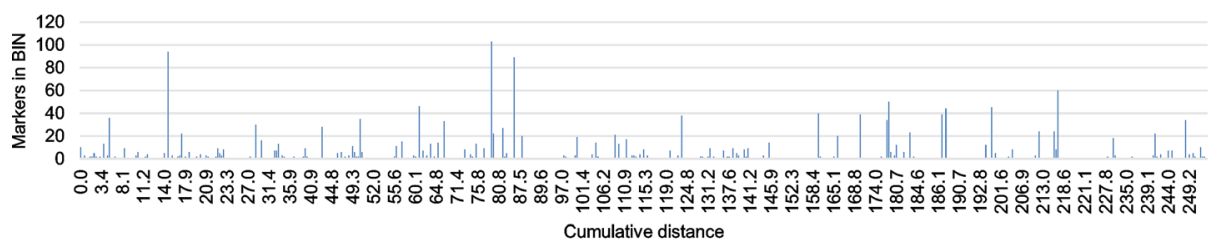

$5 R$

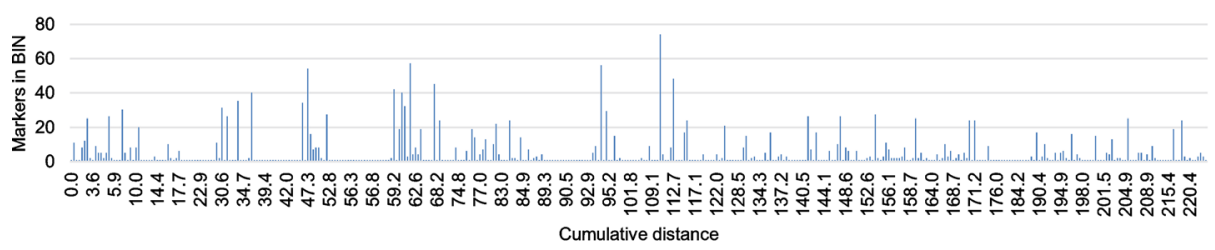

$6 R$

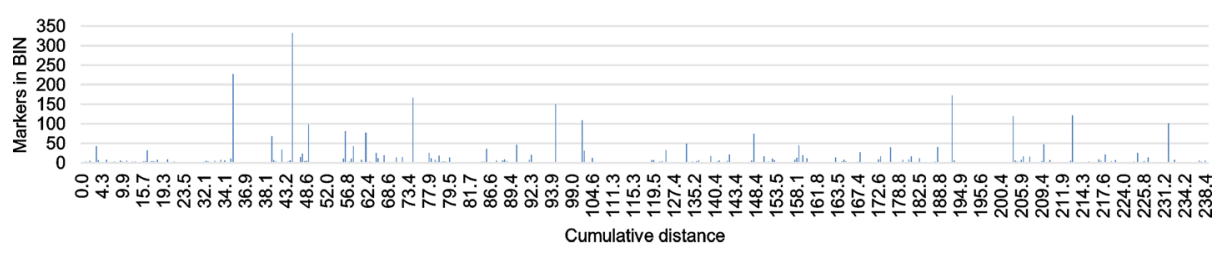

$7 R$

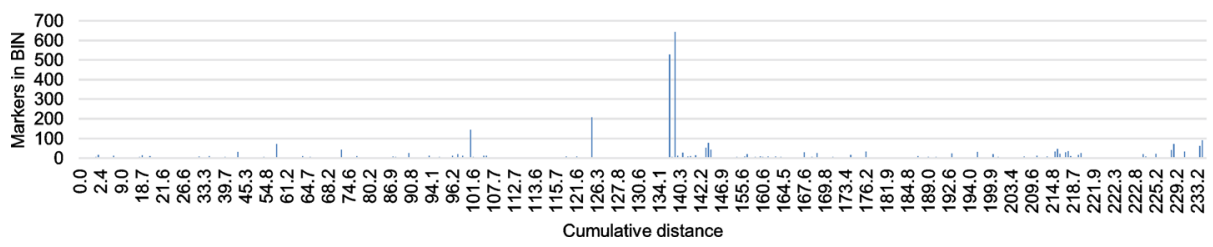




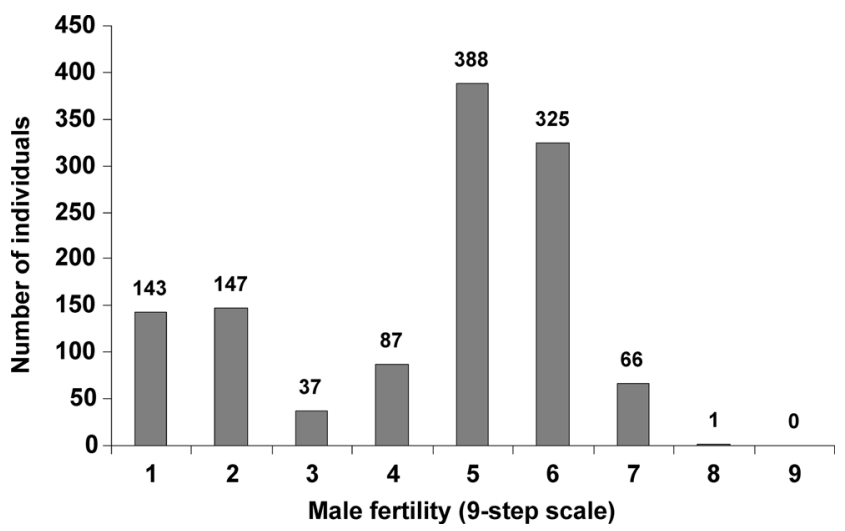

Fig. 3 Distribution of male sterility/fertility within the studied mapping population $[544 \times \mathrm{Ot} 0-20] \mathrm{BC}_{5} \mathrm{~F}_{2}$

XrPt507164, XrPt507547, and XrPt508638. All these PCR markers were originally located on the genetic map as DArTs. Unfortunately, none of the markers based on DArTseq technology was successfully converted into a polymorphic PCR marker.

Analyses performed on the extended mapping population of the [544 $\times \mathrm{Ot} 0-20] \mathrm{BC}_{5} \mathrm{~F}_{2}$ cross resulted in the construction of the genetic map based on segregation analyses of 658 individuals of the mapping population which were conducted with the use of 13 PCR-based markers. Among these markers, ten were mapped within a distance not exceeding $5 \mathrm{cM}$ from the $R f c 1$ (Fig. 4). The remaining three markers were localized at a greater distance from the target gene and proved to be helpful for comparative mapping and have allowed the correct orientation of a map with higher resolution (HRMap). Six tightly linked markers with three newly converted from DArTs into PCR-based characters (XrPt507547, XrPt400382, and $\mathrm{XrPt505373)}$ were located in the proximal side of the $R f c 1$ gene. On the distal part of the 4RL chromosome, the next three new PCR-based markers were mapped, but, here, the distance between $R f_{c} l$ and the closest marker exceeded $2.5 \mathrm{cM}$

Assessment of the utility of the PCR-based markers from the HRMap in terms of the identification of non-restorer lines and elimination of restorers was performed on a set of 95 inbred lines developed from one interline cross. From the group of ten markers located within a distance not exceeding $5 \mathrm{cM}$ from $R f c 1$, seven revealed polymorphism between parental lines 541 and Ot1-3. None of these markers was perfect in the identification of genotypes of interest. On the other hand, analyses performed on the studied lines demonstrated the high efficiency of all seven tested markers in terms of the reduction of the group of 47 restorer genotypes; the number of restorers non-detected by a given marker varied from 4 to 9 (Table 3). Application of each marker for selection also led to the loss of some non-restorer lines. For the most effective markers, the number of false-negative indications did not exceed $10 \%$ (four lines from the group of 48 ). As the studied markers were located on both proximal and distal sides of the $R f c l$ gene on the HRMap, it could be predicted that simultaneous application of a combination of markers should increase the efficiency of selection. Indeed, when all seven markers were analyzed simultaneously, false-positive indications (restorers classified by MAS as non-restorers) were reduced to only one case, and the same was noticed for false-negative results; one non-restorer line was included on the basis of genotyping into a group of restorer lines. Detailed data on the genotyping of the 95 studied inbred lines are presented in Table ESM5.

Available DNA sequences of DArT and DArTseq markers used for mapping the $R f c l$ gene (Fig. 4) were searched within databases in order to detect relationships with known gene sequences. The results of detected annotations are presented in the ESM6 file. For the majority of analyzed sequences, no relationships with sequences deposited in databases were found. Within the remaining markers, similarity to genomic sequences of wheat and Brachypodium distachyon was revealed. The functions of the indicated genes originating from these grass species do not seem to be related to male fertility restoration in rye.

\section{Discussion}

The first genetic map of all seven rye chromosomes was established more than 20 years ago, mainly based on restriction fragment length polymorphism (RFLP) markers (Devos et al. 1993). Subsequently, different marker systems have been utilized for mapping rye chromosomes, for example, RAPD (Masojć et al. 2001; Milczarski et al. 2007; Stojałowski et al. 2009), AFLP (Bednarek et al. 2003), and SSR (Hackauf and Wehling 2003; Khlestkina et al. 2004; Stojałowski et al. 2009; Myśków et al. 2010) markers, but only the application of DArT - the first available high-throughput method of genotyping S. cereale - created the possibility to construct high-density genetic maps of this species (BolibokBragoszewska et al. 2009; Milczarski et al. 2011). Sequence analyses of RNA generated by Haseneyer et al. (2011) provided the opportunity for the development of the next highthroughput marker technology: the SNP arrays successfully used for the study of the structure of the rye genome as well as for genetic mapping (Martis et al. 2013). NGS methods were recently used for the development of GBS technologies that have led to the highly efficient and cost-effective

Fig. 4 Comparative mapping of the genomic region containing $R f c 1$, the gene that restores male fertility in rye with CMS-C (the scale on the left margin shows distances in cM; markers commonly mapped within both studied mapping populations are printed in red; HRMap: genetic map based on analyses of extended numbers of individuals from the [544 $\times$ $\mathrm{Ot} 0-20] \mathrm{BC}_{5} \mathrm{~F}_{2}$ mapping population; more details are given in the text) 
RIL-S

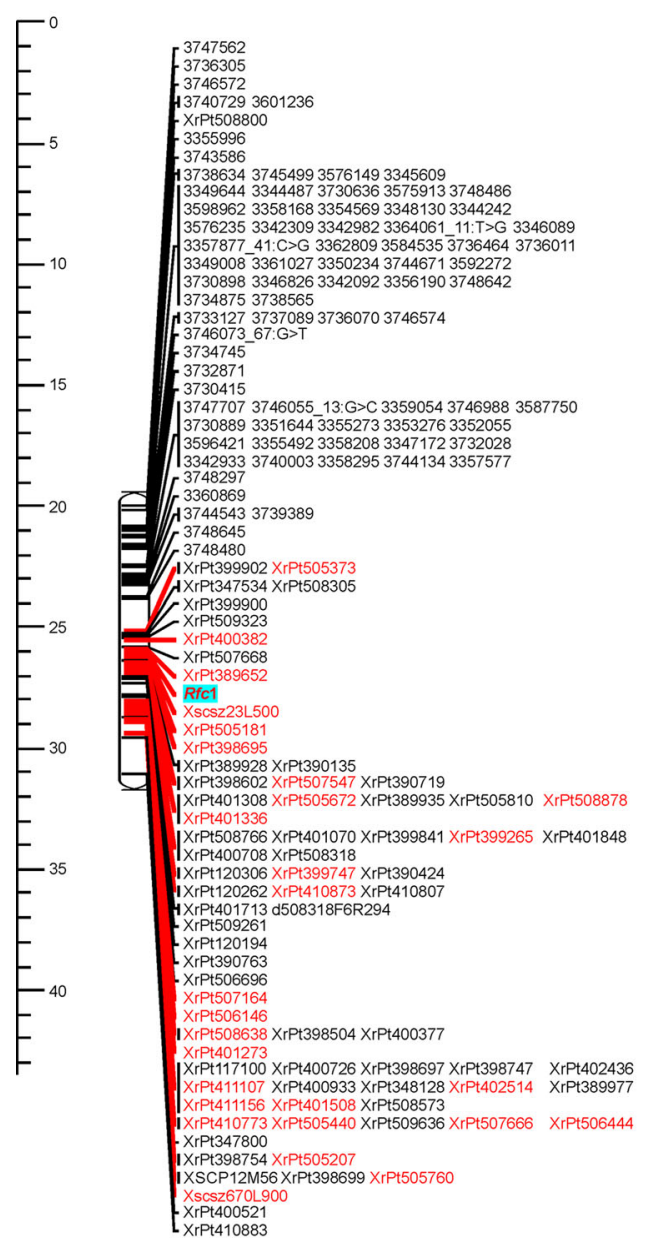

[544CxOt0-20]BC5F2

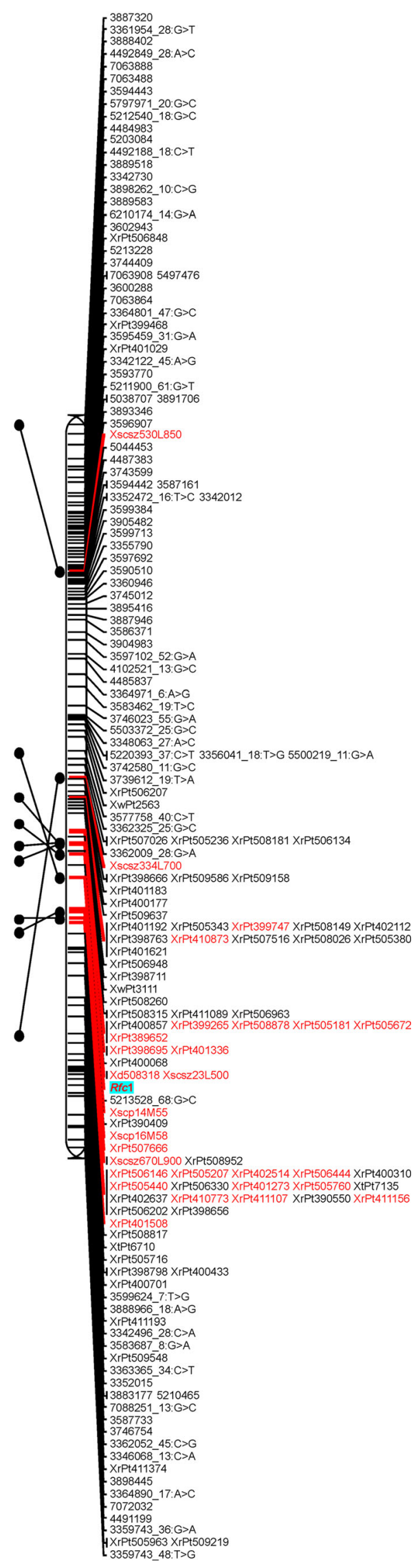


Table 3 Identification of nonrestorers and restorers for the CMS-C system within the total population of recombinant inbred lines derived from the hybrid 541 $\times$ Ot1-3 and after selection with the use of the mentioned molecular markers (significance of marker-assisted selection assessed by the $\mathrm{Chi}^{2}$ test)

\begin{tabular}{lllll}
\hline Marker & Non-restorer & Restorer & Total & Significance of MAS $\left(\mathrm{Chi}^{2}\right)$ \\
\hline XrPt411156 & 44 & 6 & 50 & $28.09^{* *}$ \\
XrPt507164 & 44 & 5 & 49 & $30.23^{* *}$ \\
XrPt508638 & 44 & 5 & 49 & $30.23^{* *}$ \\
XrPt400382 & 41 & 4 & 45 & $29.65^{* *}$ \\
XrPt505373 & 41 & 4 & 45 & $29.65^{* *}$ \\
Xscsz23L500 & 29 & 7 & 36 & $12.99^{*}$ \\
Xscp14M55 & 33 & 9 & 42 & $13.22^{*}$ \\
Total population & 48 & 47 & 95 & \\
\hline
\end{tabular}

*Statistically significant at $p=0.0005$

**Statistically significant at $p=0.0001$ discovery of SNPs in different species (Kumar et al. 2012). The high numbers of SNPs identified during a single analysis can be applied for precise assessment of genetic diversity, construction of linkage maps, and detection of markers linked with genes controlling important agronomic traits. The DArTseq platform used in this study collates the sequence analysis results in two sets of markers: dominant SilicoDArT and co-dominant SNP markers. The first group is more numerous, the second more informative (allowing for distinguishing heterozygotes from dominant homozygotes and indicating the exact character and position of mutations within the DNA molecule). Recently, GBS markers generated by this platform have been successfully applied for the construction of genetic maps of Brassica carinata (Zou et al. 2014), watermelon (Ren et al. 2015), and wheat ( $\mathrm{Li}$ et al. 2015). It should be stressed, however, that alternative methods of GBS have also been developed and have proven their effectiveness, for example, in mapping cereal genomes, such as barley and wheat (Poland et al. 2012) or rubber tree (Pootakham et al. 2015).

During genetic map construction, one of the supposed advantages of the application of SNP markers discovered by NGS technologies is the high polymorphism relatively evenly distributed within the whole genome (Kumar et al. 2012). Application of this method for extending the genetic map of the rye genome confirms this suggestion. Implementation of GBS markers (Silico-DArTs and DArTseq SNPs) into the genetic map of rye (Milczarski et al. 2011) has resulted in a significant enhancement of map density and elimination of gaps exceeding the distance of $5 \mathrm{cM}$ (Fig. 1; Table ESM1). This agrees with the results of Wendler et al. (2014), who reported the efficiency of GBS technology for the precise identification of even exceptionally small introgressions from wild into cultivated species. These authors performed their experiments on one of the most important and thoroughly studied self-fertile cereals, barley, but suggested that their statements should be of universal character and applicable for breeding activities in any crop.

The results from the localization of the $R f c l$ gene on the long arm of the $4 \mathrm{R}$ chromosome (Fig. 4) were compared with data from a newly developed mapping population [544C $\times$ $\mathrm{Ot} 0-20] \mathrm{BC}_{5} \mathrm{~F}_{2}$. The cross combination between inbred lines $544 \mathrm{C}$ and Ot0-20 has previously been used for the identification of RAPD markers linked to restorer genes (Stojałowski et al. 2004), and a high level of pollen shedding within the studied $\mathrm{F}_{2}$ population was noticed. In the currently analyzed population, the genetic diversity was significantly reduced by several cycles of back-crossing. This resulted in a lower frequency of polymorphic markers (Table 1), but due to multiple recombination events in the region containing the $R f c l$ gene, this increased the precision of mapping. Phenotypic variation within this population was relatively narrow; the most malefertile plants did not occur (Fig. 3). The lower pollen shedding by genetically fertile plants was probably due to inbreeding depression; plants of the studied population were genetically and phenotypically similar to maternal line $544 \mathrm{C}$. An additional factor that could have led to a lack of fully fertile plants within the population was elimination during back-crossing of additional, less efficient, and still unrecognized restorer genes (Stojałowski et al. 2004). Compared linkage maps from the fragment of the 4RL chromosome were very well adjusted in both the RIL-S and [544C $\times \mathrm{Ot0}-20] \mathrm{BC}_{5} \mathrm{~F}_{2}$ populations. Surprisingly, no common marker was identified from the DArTseq platform. All common markers belonged to the microarray DArT group and PCR-based markers. On the other hand, the compared linkage maps were relatively short, with limited numbers of markers, and it has been observed for three wheat mapping populations that the DArTseq platform frequently generates markers which are polymorphic within a single population ( $\mathrm{Li}$ et al. 2015). Conversion of DArT and DArTseq markers into PCR-based markers was only partly successful. PCR primers designed on the basis of short known sequences of DArTseq markers produced exclusively monomorphic amplification products in this experiment. From our other research (not published), we know that sequence data obtained during GBS analyses sometimes allow for designation of primers revealing polymorphism within mapping populations, but the frequency of such cases is low. Available sequences of DArT clones were more efficient for designation 
of PCR-based markers; about $30 \%$ of these proved to be useful for mapping and MAS.

Rye is still being extensively studied in genetic research. The first high-density maps started to be published at the end of the first decade of the 21st century (Bolibok-Bragoszewska et al. 2009; Milczarski et al. 2011) and were based on mapping populations with a limited number of analyzed genotypes not exceeding the range of 150 . Recently published results of mapping the $R f p 1$ gene that restores male fertility in the Pampa sterilizing cytoplasm were achieved with the use of 506 plant genotypes (Hackauf et al. 2012). A similar number of individuals (658) was used for the development of our HRMap. An increased number of genotyped plants resulted in some rearrangements of the map order. Of these, the most significant was the change of the position of the Xscsz334L700 marker. Its "floating" position on the map of population [ $544 \mathrm{C} \times \mathrm{Ot} 0-20] \mathrm{BC}_{5} \mathrm{~F}_{2}$ could be a result of mapping algorithms and the fact that the $R f c l$ locus and the majority of markers located on the map near this gene are dominant markers segregating, in the so-called "coupling phase", dominant alleles originating from the paternal Ot0-20 line. PCR products of the Xscsz334L700 marker originate from the maternal $544 \mathrm{C}$ line, so this marker remains in "repulsion phase" to almost all loci of the HRMap. Interestingly, one of the PCR markers, Xscp14M55, developed by Stracke et al. (2003), is present on our HRMap and a genetic map with the $R f p 1$ gene investigated by Hackauf et al. (2012). On both genetic maps, it is located proximally from the main restorer genes interacting with two genetically different cytoplasms, indicating that $R f p l$ and $R f c l$ are located in the same region of the 4RL chromosome.

The potential applicability of PCR-based markers localized in the vicinity of the $R f c l$ gene for the selection of non-restorer genotypes seems to be high. All markers located close to the target gene were statistically effective. Of course, the precision of selection by using any studied marker is not perfect and, ultimately, traditional phenotyping is still needed, but frequencies of non-restorers and restorers can be significantly changed before this time and labor-intensive activities are initiated. The efficiency of MAS can be additionally increased by the use of 2-3 markers simultaneously. On the other hand, one should stress that male fertility restoration in the CMS-C system remains under the control of more than one gene (Stojałowski et al. 2004) and development of perfect molecular markers for the selection of non-restorer or restorer genotypes needs the identification of additional, less effective restorer genes present in the rye genome. In the population [ $544 \mathrm{C} \times \mathrm{Ot} 0-20] \mathrm{BC}_{5} \mathrm{~F}_{2}$, in spite of five cycles of back-crossing, heterogeneous regions were found not only on $4 \mathrm{R}$, but also on $2 \mathrm{R}, 6 \mathrm{R}$, and $7 \mathrm{R}$ chromosomes. However, these three chromosomes are probably not engaged in the control of male fertility in the studied CMS system. Restorer genes have never been localized on chromosomes $2 R$ and $7 R$. Only on $6 R$ it was suggested the presence of some weak restorers for CMS-P (Miedaner et al. 2000; Bednarek et al. 2002) and CMS-
C (Stojałowski et al. 2004), but their localizations were not certain.

In conclusion, the genetic map of the rye genome with the $R f c 1$ gene localized on the 4RL chromosome reported here reveals the power of high-throughput marker technologies based on microarrays and NGS applications for mapping important genes. The results of mapping the $R f_{c} l$ gene indicate that this gene is located in the vicinity of the $R f p 1$ gene that restores male fertility in rye with genetically different CMSPampa (Hackauf et al. 2012).

However, it should be mentioned that high-density genetic maps presented in this study are not true high-resolution maps. The relatively low numbers of individuals genotyped within both studied mapping populations still does not allow for the construction of highly precise maps. Especially in the RIL-S population, which consisted of less than 100 individuals, this resulted in the presence of many bins containing numerous markers located at the same locus (Fig. 2). On the other hand, to our knowledge, this is the first report concerning the application of GBS markers (obtained from the DArTseq platform) for mapping rye chromosomes, and even if the resolution of the map is not very high, the presented database of markers anchored to particular chromosomes should significantly facilitate utilization of this high-throughput marker technology for further study of the rye genome.

Acknowledgments Valentina Dimitrova Ivanova and Mihail Emilov Angelov are greatly thanked by the authors for their excellent technical assistance.

Compliance with ethical standards This article does not contain any studies with human participants or animals performed by any of the authors.

Funding This study was funded by the Polish National Science Center (grant no. NN310 724540).

Conflict of interest The authors declare that they have no conflict of interest.

Open Access This article is distributed under the terms of the Creative Commons Attribution 4.0 International License (http:// creativecommons.org/licenses/by/4.0/), which permits unrestricted use, distribution, and reproduction in any medium, provided you give appropriate credit to the original author(s) and the source, provide a link to the Creative Commons license, and indicate if changes were made.

\section{References}

Adolf K, Winkel A (1985) A new source of spontaneous sterility in winter rye - preliminary results. In: Proceedings of Eucarpia Meeting of the Cereal Section on Rye, Svalöv, Sweden, June 1985, pp 293-306

Bednarek PT, Lewandowska R, Kubicka H, Masojć P (2002) Linkage groups and the indirect chromosome location of cms-P-linked AFLPs. Cell Mol Biol Lett 7:721-736 
Bednarek PT, Masojć P, Lewandowska R, Myśków B (2003) Saturating rye genetic map with amplified fragment length polymorphism (AFLP) and random amplified polymorphic DNA (RAPD) markers. J Appl Genet 44(1):21-34

Bolibok-Bragoszewska H, Heller-Uszyńska K, Wenzl P, Uszyński G, Kilian A, Rakoczy-Trojanowska M (2009) DArT markers for the rye genome - genetic diversity and mapping. BMC Genomics 10:578

Börner A, Korzun V, Polley A, Malyshev S, Melz G (1998) Genetics and molecular mapping of a male fertility restoration locus $(R f g l)$ in rye (Secale cereale L.). Theor Appl Genet 97:99-102

Cavanagh CR, Chao SM, Wang SC, Huang BE, Stephen S, Kiani S, Forrest K, Saintenac C, Brown-Guedira GL, Akhunova A, See D, Bai GH, Pumphrey M, Tomar L, Wong D, Kong S, Reynolds M, da Silva ML, Bockelman H, Talbert L, Anderson JA, Dreisigacker S, Baenziger S, Carter A, Korzun V, Morrell PL, Dubcovsky J, Morell MK, Sorrells ME, Hayden MJ, Akhunov E (2013) Genome-wide comparative diversity uncovers multiple targets of selection for improvement in hexaploid wheat landraces and cultivars. Proc Natl Acad Sci U S A 110:8057-8062

Conesa A, Götz S, García-Gómez JM, Terol J, Talón M, Robles M (2005) Blast2GO: a universal tool for annotation, visualization and analysis in functional genomics research. Bioinformatics 21:3674-3676

Devos KM, Atkinson MD, Chinoy CN, Francis HA, Harcourt RL, Koebner RMD, Liu CJ, Masojć P, Xie DX, Gale MD (1993) Chromosomal rearrangements in the rye genome relative to that of wheat. Theor Appl Genet 85:673-680

Geiger HH (2007) Strategies of hybrid rye breeding. Vortr Pflanzenzüchtg 71:1-5

Geiger HH, Morgenstern K (1975) Angewandt-genetische Studien zur cytoplasmatischen Pollensterilität bei Winterroggen. Theor Appl Genet 46:269-276 (in German)

Geiger HH, Schnell FW (1970) Cytoplasmic male sterility in rye (Secale cereale L.). Crop Sci 10:590-593

Hackauf B, Wehling P (2003) Development of microsatellite markers in rye: map construction. Plant Breed Seed Sci 48:143-151

Hackauf B, Korzun V, Wortmann H, Wilde P, Wehling P (2012) Development of conserved ortholog set markers linked to the restorer gene Rfpl in rye. Mol Breeding 30:1507-1518

Haseneyer G, Schmutzer T, Seidel M, Zhou R, Mascher M, Schön CC, Taudien S, Scholz U, Stein N, Mayer KFX, Bauer E (2011) From RNA-seq to large-scale genotyping-genomics resources for rye (Secale cereale L.). BMC Plant Biol 11:131

Khlestkina EK, Than MHM, Pestsova EG, Röder MS, Malyshev SV, Korzun V, Börner A (2004) Mapping of 99 new microsatellitederived loci in rye (Secale cereale L.) including 39 expressed sequence tags. Theor Appl Genet 109:725-732

Kobyljanskij VD (1969) About genetics of cytoplasmic male sterility in winter rye (K genetike citoplazmatičeskoj mužskoj steril'nosti u ozimoj rži). Genet Moskva 5(9):43-47 (in Russian)

Kumar S, Banks TW, Cloutier S (2012) SNP discovery through nextgeneration sequencing and its applications. Int J Plant Genomics. Article ID: 831460,15 pp

Łapiński M (1972) Cytoplasmic-genic type of male sterility in Secale montanum Guss. Wheat Inform Serv 35:25-28

Łapiński M, Stojałowski S (2003) Occurrence and genetic identity of male sterility-inducing cytoplasm in rye (Secale spp.). Plant Breed Seed Sci 48:7-23

Li H, Vikram P, Singh RP, Kilian A, Carling J, Song J, Burgueno-Ferreira JA, Bhavani S, Huerta-Espino J, Payne T, Sehgal D, Wenzl P, Singh S (2015) A high density GBS map of bread wheat and its application for dissecting complex disease resistance traits. BMC Genomics 16:216

Maccaferri M, Ricci A, Salvi S, Milner SG, Noli E, Martelli PL, Casadio R, Akhunov E, Scalabrin S, Vendramin V, Ammar K, Blanco A, Desiderio F, Distelfeld A, Dubcovsky J, Fahima T, Faris J, Korol A,
Massi A, Mastrangelo AM, Morgante M, Pozniak C, N'Diaye A, Xu S, Tuberosa R (2014) A high-density, SNP-based consensus map of tetraploid wheat as a bridge to integrate durum and bread wheat genomics and breeding. Plant Biotechnol J. doi:10.1111/pbi.12288

Madej L (1975) Research on male sterility in rye. Plant Breed Seed Sci $19: 421-422$

Martis MM, Zhou R, Haseneyer G, Schmutzer T, Vrána J, Kubaláková M, König S, Kugler KG, Scholz U, Hackauf B, Korzun V, Schön CC, Doležel J, Bauer E, Mayer KFX, Stein N (2013) Reticulate evolution of the rye genome. Plant Cell 25:3685-3698

Masojć P, Myśków B, Milczarski P (2001) Extending a RFLP-based genetic map of rye using random amplified polymorphic DNA (RAPD) and isozyme markers. Theor Appl Genet 102:1273-1279

Miedaner T, Glass C, Dreyer F, Wilde P, Wortmann H, Geiger HH (2000) Mapping of genes for male-fertility restoration in 'Pampa' CMS winter rye (Secale cereale L.). Theor Appl Genet 101:1226-1233

Milczarski P, Banek-Tabor A, Lebiecka K, Stojałowski S, Myśków B, Masojć P (2007) New genetic map of rye composed of PCR-based molecular markers and its alignment with the reference map of the DS2 $\times$ RXL10 intercross. J Appl Genet 48:11-24

Milczarski P, Bolibok-Bragoszewska H, Myśków B, Stojałowski S, Heller-Uszyńska K, Góralska M, Bragoszewski P, Uszyński G, Kilian A, Rakoczy-Trojanowska M (2011) A high density consensus map of rye (Secale cereale L.) based on DArT markers. PLoS One 6(12):e28495. doi:10.1371/journal.pone.0028495

Myśków B, Stojałowski S, Milczarski P, Masojć P (2010) Mapping of sequence-specific markers and loci controlling preharvest sprouting and alpha-amylase activity in rye (Secale cereale L.) on the genetic map of an F2 (S120×S76) population. J Appl Genet 51:283-287

Poland JA, Brown PJ, Sorrells ME, Jannink J-L (2012) Development of high-density genetic maps for barley and wheat using a novel twoenzyme genotyping-by-sequencing approach. PLoS One 7:e32253

Pootakham W, Ruang-Areerate P, Jomchai N, Sonthirod C, Sangsrakru D, Yoocha T, Theerawattanasuk K, Nirapathpongporn K, Romruensukharom P, Tragoonrung S, Tangphatsornruang S (2015) Construction of a high-density integrated genetic linkage map of rubber tree (Hevea brasiliensis) using genotyping-bysequencing (GBS). Front Plant Sci 6:367

Putt ED (1954) Cytogenetic studies of sterility in rye. Can J Agric Sci 34: 81-119

Ren R, Ray R, Li P, Xu J, Zhang M, Liu G, Yao X, Kilian A, Yang X (2015) Construction of a high-density DArTseq SNP-based genetic map and identification of genomic regions with segregation distortion in a genetic population derived from a cross between feral and cultivated-type watermelon. Mol Genet Genomics 290:1457-1470. doi:10.1007/s00438-015-0997-7

Ronin Y, Mester D, Minkov D, Belotserkovski R, Jackson BN, Schnable PS, Aluru S, Korol A (2012) Two-phase analysis in consensus genetic mapping. G3: Genes, Genomes, Genetics 2:537-549

Stojałowski S, Łapiński M, Masojć P (2004) RAPD markers linked with restorer genes for the $\mathrm{C}$-source of cytoplasmic male sterility in rye (Secale cereale L.). Plant Breed 123:428-433

Stojałowski S, Łapiński M, Szklarczyk M (2006) Identification of sterility-inducing cytoplasms in rye using the plasmotype-genotype interaction test and newly developed SCAR markers. Theor Appl Genet 112:627-633

Stojałowski S, Kociuba M, Stochmal B, Kondzioła M, Jaciubek M (2008) Determining the plasmotypic structure of rye populations by SCAR markers. J Appl Genet 49(3):229-232

Stojałowski S, Myśków B, Milczarski P, Masojć P (2009) A consensus map of chromosome 6R in rye (Secale cereale L.). Cell Mol Biol Lett 14:190-198

Stojałowski SA, Milczarski P, Hanek M, Bolibok-Bragoszewska H, Myśków B, Kilian A, Rakoczy-Trojanowska M (2011) DArT markers tightly linked with the $R f c l$ gene controlling restoration of 
male fertility in the CMS-C system in cultivated rye (Secale cereale L.). J Appl Genet 52:313-318

Stracke S, Schilling AG, Förster J, Weiss C, Glass C, Miedaner T, Geiger HH (2003) Development of PCR-based markers linked to dominant genes for male-fertility restoration in Pampa CMS of rye (Secale cereale L.). Theor Appl Genet 106:1184-1190

Van Ooijen JW, Voorrips RE (2001) JoinMap® 3.0, software for the calculation of genetic linkage maps. Plant Research International, Wageningen

Voorrips RE (2002) MapChart: software for the graphical presentation of linkage maps and QTLs. J Hered 93(1):77-78

Wang J, Li H, Zhang L, C Li, Meng L (2014a) Users' Manual of QTL IciMapping. The Quantitative Genetics Group, Institute of Crop Science, Chinese Academy of Agricultural Sciences (CAAS), Beijing 100081, China, and Genetic Resources Program, International Maize and Wheat Improvement Center (CIMMYT), Apdo. Postal 6-641, 06600 Mexico, D.F., Mexico

Wang S, Wong D, Forrest K, Allen A, Chao S, Huang BE, Maccaferri M, Salvi S, Milner SG, Cattivelli L, Mastrangelo AM, Whan A,
Stephen S, Barker G, Wieseke R, Plieske J, International Wheat Genome Sequencing Consortium, Lillemo M, Mather D, Appels R, Dolferus R, Brown-Guedira G, Korol A, Akhunova AR, Feuillet C, Salse J, Morgante M, Pozniak C, Luo M-C, Dvorak J, Morell M, Dubcovsky J, Ganal M, Tuberosa R, Lawley C, Mikoulitch I, Cavanagh C, Edwards KJ, Hayden M, Akhunov E (2014b) Characterization of polyploid wheat genomic diversity using a high-density 90000 single nucleotide polymorphism array. Plant Biotechnol J 12:787-796

Warzecha R, Salak-Warzecha K (1996) Comparative studies on CMS sources in rye. Vortr Pflanzenzüchtg 35:39-49

Wendler N, Mascher M, Nöh C, Himmelbach A, Scholz U, RugeWehling B, Stein N (2014) Unlocking the secondary gene-pool of barley with next-generation sequencing. Plant Biotechnol J 12: $1122-1131$

Zou J, Raman H, Guo S, Hu D, Wei Z, Luo Z, Long Y, Shi W, Fu Z, Du D, Meng J (2014) Constructing a dense genetic linkage map and mapping QTL for the traits of flower development in Brassica carinata. Theor Appl Genet 127:1593-1605 